\title{
Mandibular second premolar with Vertucci Type II root canal morphological system: a case report
}

\author{
Yelda Erdem Hepşenoğlu, (1) Şeyda Erşahan \\ Department of Endodontics, İstanbul Medipol University Faculty of Dentistry, İstanbul, Turkey
}

\begin{abstract}
Successful root canal treatment relies on correct access cavity preparation, sufficient cleaning, adequate shaping, and complete obturation. Prior to these, location of all canals in the tooth plays an important part in the initial treatment procedures. While most teeth have a normal morphology, we should recognize that variations do exist. Mandibular premolars have been reported with complex anatomical aberrations, making them one of the most difficult teeth to manage endodontically. A case of endodontic treatment of a mandibular second premolar with two root canals and one apical foramen (Vertucci Type II) was described. A 13-year-old female presented with a chief complaint of pain in the mandibular right second premolar. The pain disturbed her sleep and lingered for several minutes even after removal of a thermal stimulus. Clinical examination revealed tenderness to percussion in the tooth. A clinical diagnosis of irreversible pulpitis with symptomatic apical periodontitis was made and root canal therapy was performed following the standard protocols. Although the prevalence of one root and two canals in mandibular premolars is very low, the clinician should always be mindful of variations in the number of roots and canals for proper management of such cases.
\end{abstract}

Keywords: Mandibular premolar; one root; root canal treatment; two canals; Vertucci Type II root canal.

Quccessful endodontic treatment has become an im$\checkmark$ portant and effective method for maintaining and preserving dental health. A thorough understanding of the root canal morphology and correct evaluation of preoperative radiographs are essential parts of endodontic therapy. Variations in the form of aberrant canal configurations, accessory canals, bifurcation, isthmuses, and anastomoses are often difficult to identify, thus creating a problem for endodontic treatment. ${ }^{[1]}$ Failure to recognize and treat an extra canal might provide a constant source of irritation, thereby compromising the long-term success of the root canal therapy. ${ }^{[2]}$ Vertucci $^{[3,4]}$ (2005) has classified morphological patterns of the root canal systems into eight types. In the simplest form, each root has a single canal and a single apical foramen (Type I). Commonly, however, other canal complexities are present and exit the root as one, two, or more apical canals (Types II-VIII). ${ }^{[3,4]}$ In general, the mandibular second premolars have a single root and a single canal (Vertucci Type I; 99.28\% and $86.9 \%$, respectively). ${ }^{[5]}$ However, the occurrence of two root canals with one apical foramen (Vertucci Type II) in mandibular second premolars is a rare entity ranging from 7 to $9 \% .{ }^{[6,7]}$ The purpose of this case report was to present successful management of a mandibular second premolar with a rare anatomical variation, two root canals with one apical foramen (Vertucci Type II).

Correspondence: Dr. Şeyda Erşahan. İstanbul Medipol Üniversitesi Diş Hekimliği Fakültesi Hastanesi,

Birlik Mah., Bahçeler Cad., No: 5, Esenler, İstanbul, Turkey.

Tel: +90 212 - 4401000 e-mail: seydaersahan@hotmail.com

Submitted: May 16, 2018 Accepted: May 30, 2018

(C)2019 Turkish Endodontic Society

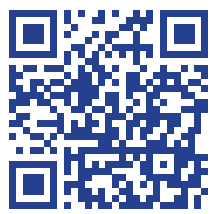




\section{Case report}

An 13-year-old female patient reported to the Department of Endodontics, Faculty of Dentistry, Istanbul Medipol University, with a chief complaint of pain in the right mandibular region. On history taking, there was the spontaneous pain, disturbing her sleep. The pain aggravates on chewing foods. Intraoral examination showed a deep disto-occlusal carious lesion in right mandibular second premolar. On clinical examination, a deep carious lesion was seen with respect to 45 and tenderness to percussion was observed in that tooth. The exaggerated response was observed during pulp testing with electric pulp tester, and lingering pain was observed with cold pulp test compared to contralateral teeth. No periradicular lesions were found to be associated with the tooth (Fig. la). A clinical diagnosis of irreversible pulpitis with symptomatic apical periodontitis was made. Root canal treatment was decided and explained to the patient. CT could not be planned.

The treatment was initiated with local anesthesia, inferior alveolar nerve block on the right side of jaw using $40 \mathrm{mg} / \mathrm{mL}$ articaine hydrochloride with $0.012 \mathrm{mg} / \mathrm{mL}$ epinephrine hydrochloride (Ultracaine D-S forte; Aventis Pharma, Istanbul, Turkey). After local anesthesia and rubber dam isolation, straight-line access was gained to the pulp chamber. Two root canals were located, one in the buccal and one in the lingual. The working lengths of the root canals were determined by using Raypex 6 (VDW, Munich, Germany) apex locator, and checked with a radiograph, which revealed two canals with one apical foramen (Fig. lb). Cleaning and shaping was performed using a crown-down technique with Revo $S$ files (Micro- Mega, Besançon, France) using 5\% sodium hypochlorite irrigation. (\# 40/.06 taper). Patency was achieved in the canals and was maintained with a $10 \mathrm{~K}$ file (DentsplyMaillefer, Ballaigues, Switzerland). Root canal filling was completed by using the cold lateral compaction method with AH Plus (Dentsply DeTrey, Konstanz, Germany) sealer and gutta-percha (Diadent Group International, Chungcheongbuk-do, Korea). The root canal treatment was completed in a single-visit appointment. The tooth was later restored with composite resin (3M, St. Paul, MN, USA). A postoperative periapical radiograph showed complete filling of the two main canals (Fig. lc). Following the completion of endodontic treatment of the right mandibular second premolar, the patient, who felt no obvious symptoms or signs.
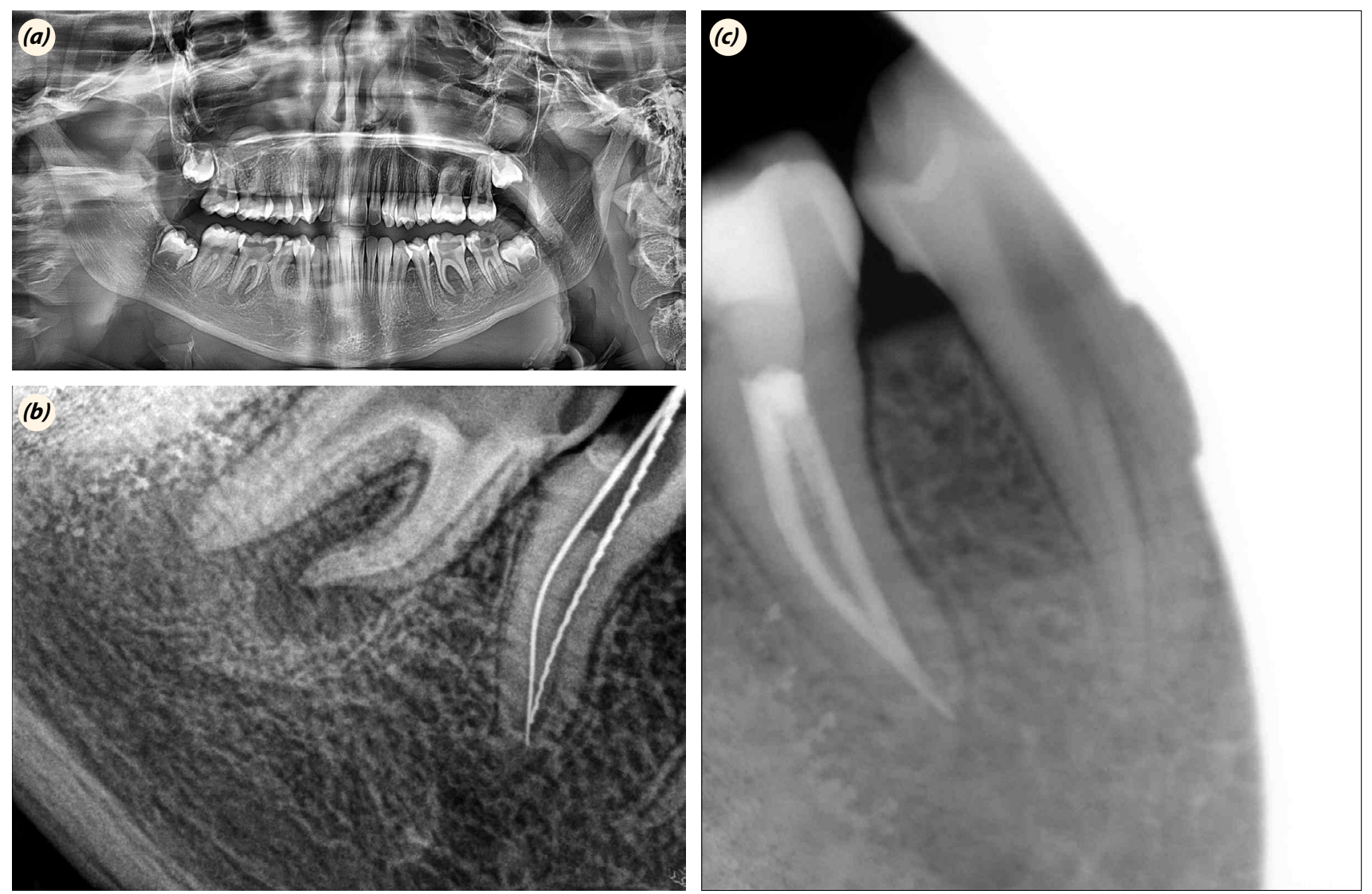

Fig. 1. (a) Diagnostic radiograph showing two canals. (b) Working length radiograph. (c) Periapical radiograph following the canals. 


\section{Discussion}

The complex anatomy of the mandibular premolar should be thoroughly understood for proper management and a better prognosis. ${ }^{[8]}$ Vertucci Type II root canal morphology in mandibular premolars has great clinical importance during endodontic treatment. These morphology, in which there are two canals with one apical foramina, poor or no filling of the lingual root canal may lead to failure of the endodontic therapy. ${ }^{[9]}$ The clinician must be recognize the anatomical morphology of a normal root canal system, as well as any variations that may exist. For this purpose, professionals must have a good knowledge of the internal anatomy of teeth and also know the radiographic techniques and their variations, in order to have a perfect visualization of root canals.

Several studies regarding the anatomical variations in mandibular premolar teeth are available in the literature. ${ }^{[5,10,11]}$ In mandibular second premolars, Vertucci Type I with single canal from orifice to apical foramen is the most prevalent canal type (90\%). ${ }^{[5]}$ Second premolars presented with a second canal in Iranian $(5.8-17.5 \%)^{[6,12,13]}$ or Jordanian $(22.8 \%)^{[14]}$ populations. However, Mongoloid ${ }^{[15,16]}$ and Hispanic ${ }^{[8]}$ populations presented a much lower incidence of a second canal, $2 \%$ and $1.2 \%$, respectively. The second-most-prevalent canal type of the mandibular second premolar was type II, according to the findings of Rahimi et al. ${ }^{[6]}$ (7.9\%, in Jordanian population), and Sikri and $\mathrm{Sikri}^{[7]}$ (9\%, in Indian population). Genetic and racial variations are factors that may affect root canal anatomy and morphology. In a Turkish sample, Sert and Bayirli[11] evaluated the proportion of different root canal types and authors reported that $7 \%$ of the mandibular second premolar teeth had Vertucci Type II root canal morphology. This case report demonstrated successful management of a mandibular second premolar with Vertucci Type II root canal morphology.

Endodontic success in teeth with additional canals requires a meticulous clinical and radiographic examination. Angled preoperative radiographs, cone beam computed tomography images (CBCT), examination of the pulp-chamber floor with a sharp explorer, troughing of the grooves with ultrasonic tips, staining the chamber floor with $1 \%$ methylene blue dye, performing the sodium hypochlorite "champagne bubble test," and visualizing canal bleeding points are all essential aids in locating the root canal orifices. ${ }^{[17]}$ The search for additional orifices is also abetted by the use of microscopes, magnifying loupes, and fiber-optic transillumination to locate the developmental line between the mesiobuccal and mesiolingual orifices. ${ }^{[18]}$ In the present case, as we were able to observe two canals by a standard periapical radiograph $\left(20^{\circ}\right.$ an- gulation from mesial and distal side), we did not need to take CBCT.

In the present study, the shape of access cavity was ovoid in buccolingual direction. We negotiated and secured canals with small-sized hand files. Once the canal was manually reproduced, a dedicated mechanical glide path file was used to expand the working width and preshape a canal in preparation for shaping procedures. Then, both buccal and lingual canals were prepared with $\mathrm{Ni}-\mathrm{Ti}$ rotary files (\# 40/.06). The clinician should be careful in instrumentation of such canals, as overzealous instrumentation can lead to strip or lateral perforations in these critical areas.

Mandibular premolars, because of their complex canal systems, are often considered the most difficult of all teeth on which to perform successful endodontic treatment. ${ }^{[11,19]}$ However, various innovations in diagnostics, magnification, operative instruments, and techniques and an updated knowledge with regard to the anatomy of mandibular premolars could certainly improve the endodontic success rates of even the most challenging cases.

\section{Conclusion}

The present case report proved the importance of knowledge of root canal system and the most common variations one must keep in his/her mind before starting an endodontic treatment of mandibular premolar teeth.

Conflict of interest: None declared.

\section{References}

1. Daokar SG, Kalekar Yadao AS, Ghunawat DB, Kakde DD. All the mandibular incisors with double canals in a single patient: A rare case. J Int Oral Health 2015;7:46-9.

2. Mohan AG, Rajesh EA, George L, Sujathan, Josy SA. Maxillary lateral incisors with two canals and two separate curved roots. Contemp Clin Dent 2012;3:519-21.

3. Vertucci FJ. Root canal anatomy of the mandibular anterior teeth. J Am Dent Assoc 1974;89:369-71.

4. Vertucci FJ. Root canal anatomy of the human permanent teeth. Oral Surg Oral Med Oral Pathol 1984;58:589-99.

5. Kottoor J, Albuquerque D, Velmurugan N, Kuruvilla J. Root anatomy and root canal configuration of human permanent mandibular premolars: a systematic review. Anat Res Int 2013;2013:254250.

6. Rahimi S, Shahi S, Yavari HR, Manafi H, Eskandarzadeh N. Root canal configuration of mandibular first and second premolars in an Iranian population. J Dent Res Dent Clin Dent Prospects 2007;1:59-64.

7. Sikri VK, Sikri P. Mandibular premolars: aberrations in pulp space morphology. Indian J Dent Res 1994;5:9-14. 
8. Pineda F, Kuttler Y. Mesiodistal and buccolingual roentgenographic investigation of 7,275 root canals. Oral Surg Oral Med Oral Pathol 1972;33:101-10.

9. Yoshioka T, Villegas JC, Kobayashi C, Suda H. Radiographic evaluation of root canal multiplicity in mandibular first premolars. J Endod 2004;30:73-4.

10. Calişkan MK, Pehlivan Y, Sepetçioğlu F, Türkün M, Tuncer SS. Root canal morphology of human permanent teeth in a Turkish population. J Endod 1995;21:200-4.

11 . Sert S, Bayirli GS. Evaluation of the root canal configurations of the mandibular and maxillary permanent teeth by gender in the Turkish population. J Endod 2004;30:391-8.

12. Hasheminia M, Hashemi A. Frequency of canal configuration in maxillary first premolars and mandibular second premolars. J Isfahan Dent School 2005;1:59-64.

13. Rahimi S, Shahi S, Yavari HR, Reyhani MF, Ebrahimi ME, Rajabi E. A stereomicroscopy study of root apices of human maxillary central incisors and mandibular second premolars in an Iranian population. J Oral Sci
2009;51:411-5.

14. Awawdeh LA, Al-Qudah AA. Root form and canal morphology of mandibular premolars in a Jordanian population. Int Endod J 2008;41:240-8.

15. Tian YY, Guo B, Zhang R, Yu X, Wang H, Hu T, et al. Root and canal morphology of maxillary first premolars in a Chinese subpopulation evaluated using cone-beam computed tomography. Int Endod J 2012;45:996-1003.

16. Miyoshi S, Fujiwara J, Tsuji Y Nakata T, Yamamoto K. Bifurcated root canals and crown diameter. J Dent Res 1977;56:1425.

17. Vertucci FJ. Root canal morphology and its relationship to endodontic procedures. Endodontic Topics 2005;10:3-29.

18. Vertucci FJ, Haddix JE. Tooth morphology and access cavity preparation. In: Hargreaves KM, Cohen S, editors. Pathways of the Pulp. 10th ed. St. Louis: Mosby Inc; 2002. p. 136-222.

19. Slowey RR. Root canal anatomy. Road map to successful endodontics. Dent Clin North Am 1979;23:555-73. 